\title{
Risk Factors of Multi-Drug Resistant Tuberculosis in Addis Ababa, Ethiopia: A Matched Case-Control Study
}

\author{
Meseret A. Deressa, Meaza Demissie \\ Addis Continental Institute of Public Health \& Haramaya University, Addis Ababa, Ethiopia \\ Email: messi96@yahoo.com
}

Received 8 April 2014; revised 18 May 2014; accepted 26 June 2014

Copyright (C) 2014 by authors and OALib.

This work is licensed under the Creative Commons Attribution International License (CC BY). http://creativecommons.org/licenses/by/4.0/

\begin{abstract}
Objective: This study determined risk factors associated with MDR-TB among MDR TB/HIV coinfected cases and TB/HIV co-infected controls attending ART clinics in Addis Ababa. Methods: A health facility based 1:3 matched case-control study was conducted from January to February 2013. Study subjects were selected using multistage probability sampling. Cases were matched to controls using age, sex and HIV sero-positive status. Bivariate and multivariate conditional logistic regression models were used to determine risk factors associated with MDR-TB. Results: A total of 96 cases were matched to 288 controls. Risk factors for MDR-TB were found to be: previous TB treatment $(A O R=5.7,95 \% \mathrm{CI}: 1.82-8.32)$, having opportunistic infection $(\mathrm{AOR}=2.23,95 \% \mathrm{CI}$ : 1.27 - 3.92), primary education ( $A O R=1.37,95 \%$ CI: 1.08 - 1.74), unemployment $(A O R=3.44,95 \%$ CI: 1.82 - 6.48), long distance of the health facility (AOR = 10.18, 95\% CI: 1.22 - 84.77), $1 \mathrm{hr}-24 \mathrm{hrs}$ to reach the health facility (AOR $=2.19,95 \%$ CI: $1.15-4.15)$ and $>24 \mathrm{hrs}$ to reach the health facility (AOR = 4.56, 95\% CI: 1.14 - 18.25). Conclusion and Recommendation: The identified risk factors should be given priority by TB control programs under Addis Ababa health bureau to control MDR-TB. In addition, these findings need to be corroborated with multi-centre case-control studies to bring out nationally relevant risk factors for MDR-TB.
\end{abstract}

\section{Keywords}

MDR-TB, Tuberculosis, Risk, Case-Control, HIV

Subject Areas: Infectious Diseases, Public Health 


\section{Introduction}

Multidrug-resistant (MDR) and extensively drug-resistant (XDR) tuberculosis (TB) continue to emerge in high HIV prevalence settings, and their mortality in HIV co-infected patients remains high [1]-[5]. Each year, globally, about 440,000 MDR-TB cases are estimated to emerge, and 150,000 people with MDR-TB die [6]. Ethiopia ranks seventh among the 22 high tuberculosis burden countries and fifteenth in the list of 27 countries with the highest number of estimated multidrug-resistant tuberculosis (MDR-TB) cases [7].

An estimated 5000 MDR TB cases exist in Ethiopia [8]. Studies done in Addis Ababa by Demisse et al. (2005) [9] and Abate et al. (2006) [10] found the prevalence of MDR-TB as $7.2 \%$ and $12 \%$ respectively among retreated TB patients. Another study done two years later by Meskel et al. (2008) revealed that $26.2 \%$ of the isolates were MDR-TB and concluded that MDR-TB was an emerging problem among re-treatment cases of pulmonary TB in Addis Ababa [11].

However, to our knowledge, no study was done to assess risk factors for Multi drug resistant tuberculosis targeting MDR-TB/HIV and TB/HIV patients. Therefore, this study determined risk factors for MDR-TB among MDR-TB/HIV co-infected cases and TB/HIV co-infected controls in Addis Ababa.

\section{Methodology}

\subsection{Study Design and Study Area}

Matched (1:3) case-control study was conducted from January to February 2013. The study was conducted in Addis Ababa, Ethiopia. Administratively, Addis Ababa is divided in to 10 sub cities and 116 woredas with an area of 540 sq. km [12]. An estimated 210,306 people live with HIV in Addis Ababa with TB/HIV co-infection rate of 33\% according to the Federal Ministry of Health Report [13] [14]. There are 33 ART Clinics in Addis Ababa of which 24 are in health centers and 9 are in hospitals [15].

\subsection{Study Population}

Case patients were patients with HIV who had laboratory confirmed isolates resistant at least to isoniazid and rifampin and had been hospitalized in the ART ward or seen in the ART clinic. Control patients were patients with TB/HIV infection that were hospitalized in the ART ward or seen in the ART clinic following TB treatment for more than two months. In addition, they had confirmed sputum smear conversion at the end of the second month to be certain the infection had not transformed to MDR-TB. Exclusion criteria for cases and controls: Patients $<18$ years old, unable to hear, mentally retarded, seriously ill and who visited the health facility for more than once.

\subsection{Sample Size}

The sample size for the case control study was based on two population proportion formula with an assumption of $80 \%$ power $(1-\beta), 5 \%$ type I error at $95 \%$ Confidence Interval (CI) and odds ratio of at least 2 in the target population.

\subsection{Sampling Procedure}

Multistage probability sampling method was used to select the study participants which included simple random sampling to select primary sampling units, stratified sampling to ensure proportional allocation and systematic random sampling to select individual study subjects consecutively (see Figure 1 and Figure 2). Data collection was started from the $2^{\text {nd }}$ client (randomly selected from 1 - 9) on January $1^{\text {st }}$ in every of the selected health facilities. Then after, every $19^{\text {th }}$ person of PLW TB/HIV $(\mathrm{N} / \mathrm{n}=5513 / 288)$ and every $7^{\text {th }}$ person of people living with (PLW) MDR-TB/HIV ( $/ \mathrm{n}=663 / 96)$ was assigned to be a selected respondent. Matching a case to 3 controls by age (frequency matching), sex and HIV sero-positive status was done to increase the power of the study. The person next to the $19^{\text {th }}$ person of PLW TB/HIV or next to the $7^{\text {th }}$ person of PLW MDR-TB/HIV was assigned to be selected in case of a non-response.

\subsection{Data Collection}

The data collection included obtaining secondary data from a card containing patient's history (drug susceptibil- 


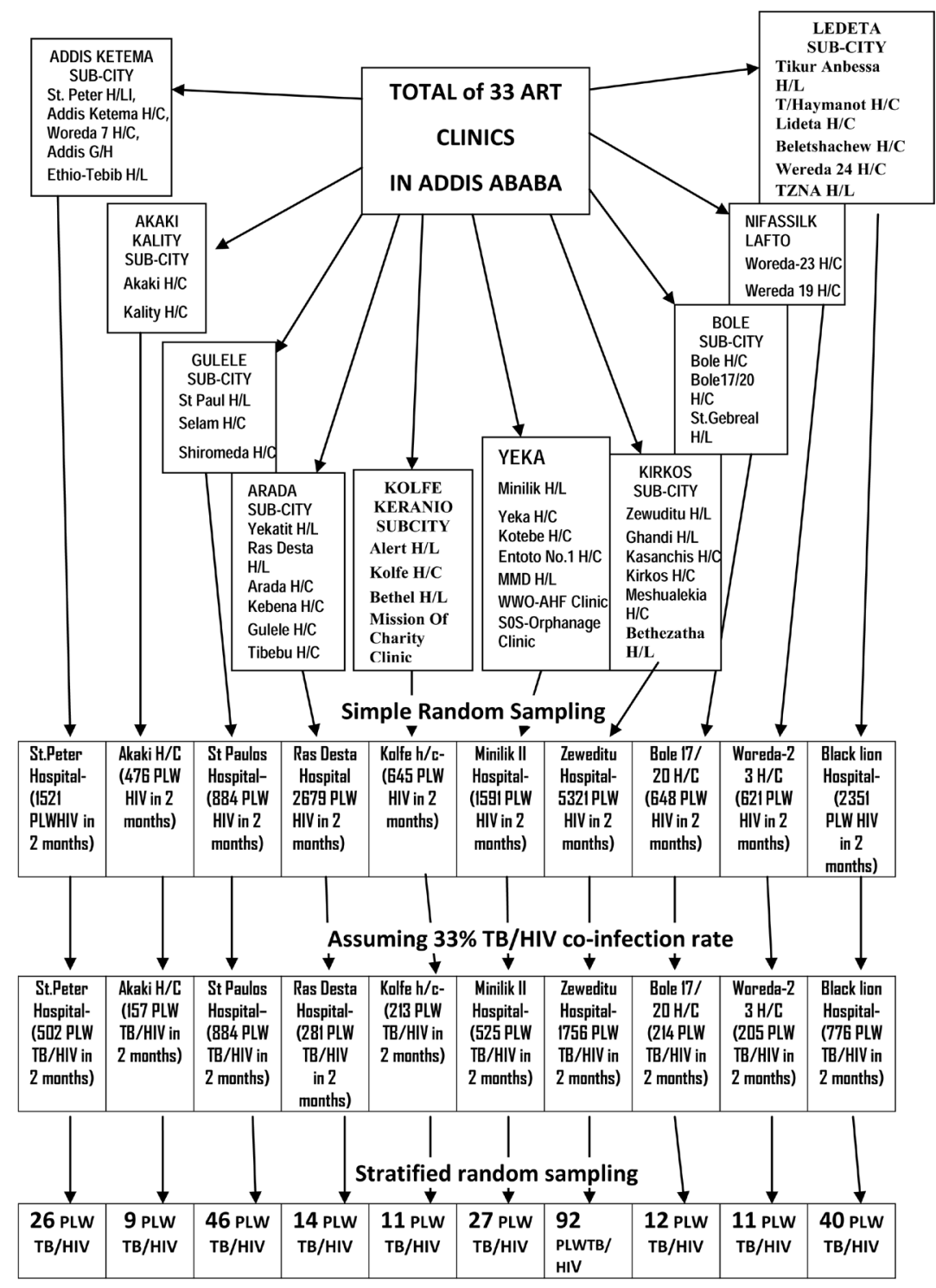

Figure 1. Multistage sampling procedure of the study for controls.

ity testing result, CD4 count, sputum smear result, stage of treatment etc.) and face to face interview about knowledge of MDR-TB and associated factors using an Amharic version structured questionnaire by 10 trained nurses who worked in the selected ART clinics. To maintain the data quality, onsite training was given for the10 interviewers (nurses) for two days on sampling procedure, inclusion criteria, ethical consideration, data collection procedure, how to approach clients, and discussion of every question to avoid ambiguity on some questions. In addition, the questionnaire was first translated to Amharic and then back to English and pre-tested 1 week before the actual data collection at Menelik II Hospital on 5\% of the sample size i.e. 19 ART clinic attendants with TB/HIV co-infection. Moreover, timely supervision and checking of data completion was made by the supervisors every day. The principal investigator was also working as a co-supervisor in each health facility.

\subsection{Statistical Analysis}

Data entry and cleaning was done by a trained encoder using Epi-info (Center for Disease Control and Prevention Atlanta, GA) version 3.5.1 and descriptive statistical analysis was made in SPSS (Statistical Package for Social Science) version 15. The descriptive statistics for knowledge of MDR-TB was computed by considering overall knowledge score $\geq 5.39$ as good knowledge of MDR-TB and score $<5.39$ as poor MDR-TB knowledge 


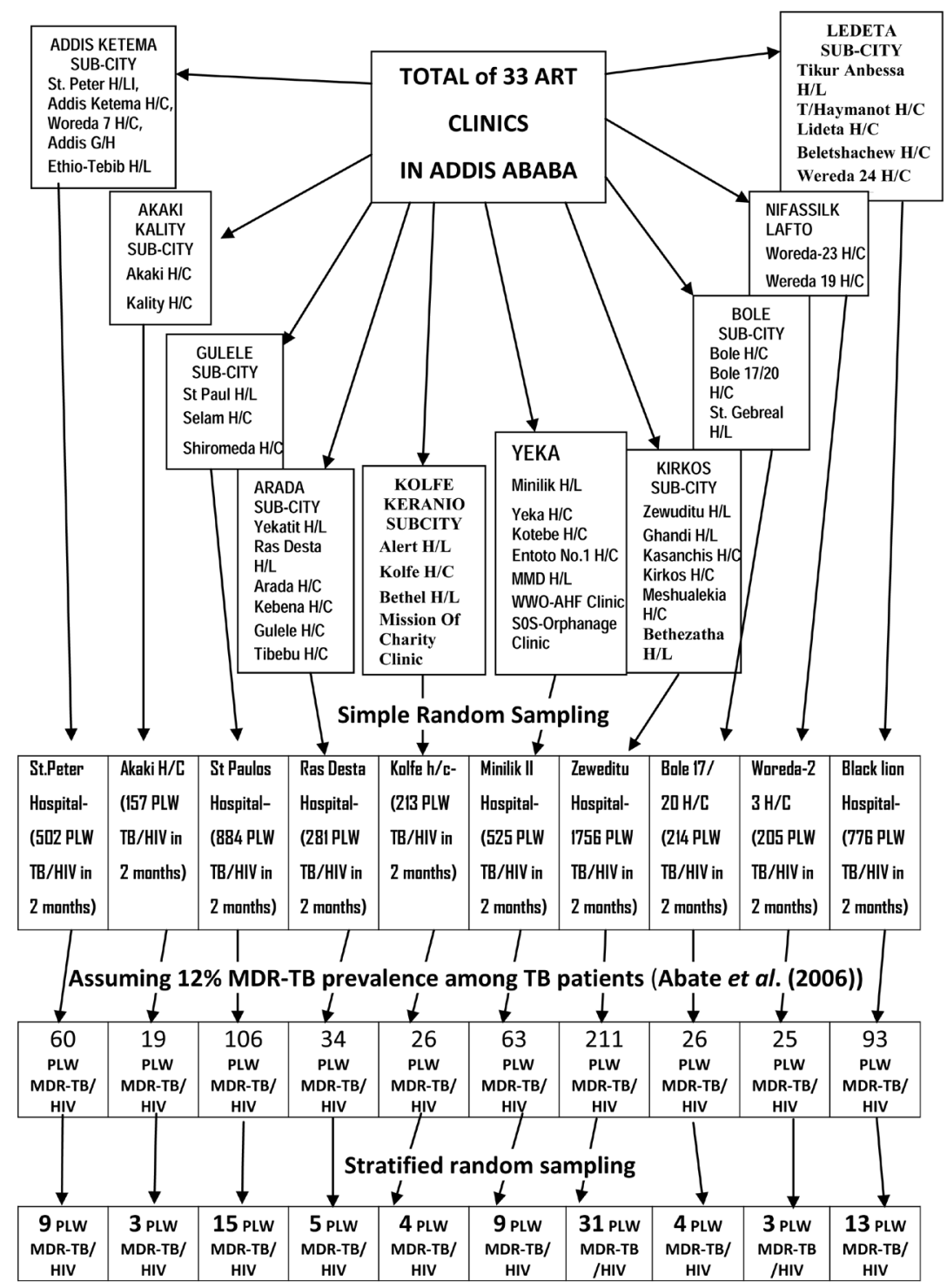

Figure 2. Multistage sampling procedure of the study for cases.

(see operational definition). Conditional logistic regression using Epi-info version 3.5.1. was used to determine risk factors associated with MDR-TB and calculate $P$-values $(P)$, Odds ratios $(O R s)$ and $95 \%$ confidence intervals (CIs). A 1:3 matched bivariate conditional logistic regression analysis was first made and, step wisely, variables with $P$-value $<0.2$ in the bivariate test were included in the multivariate conditional logistic regression analysis to adjust for the effect of confounding variables. Variables statistically significant $(P<0.05)$ in the multivariate conditional logistic regression analysis were considered as risk factors for MDR-TB.

\subsection{Ethical Consideration}

Ethical clearance was obtained from Haramaya University and Addis Continental Institute of Public Health ethical review board. Permission to conduct the study was also obtained from Addis Ababa Regional Health Bureau and each selected health facility. When the study was conducted ethical clearance was also obtained from the health facilities on behalf of the study participants to collect a secondary data and to trace the study participants who were not available at the clinic during the study period. Prior to the administration of the questionnaire, verbal and written informed consent was obtained from the study participants after a brief explanation of the benefit of the study. 


\section{Result}

\subsection{Socio-Demographic Characteristics of Study Participants}

A total of 384 matched study subjects participated in the study of which 96 were cases and 288 were controls with $100 \%$ response rate. Males' contribution was $57.2 \%$ in the study while females' contribution accounts $42.7 \%$. The mean age of the study subjects were 31.42 years $( \pm 9.05 \mathrm{SD})$ for controls and 31.39 years $( \pm 9.12 \mathrm{SD}$ ) for cases while the age range was from 18years to 62 years for both cases and controls. The majority of the cases (72\%) and controls (86.1\%) had Orthodox religion. Of the controls $48.3 \%$ had a level of secondary education and above where as the majority of the cases attended primary education. Regarding employment status, 77 (80.2\%) of the cases and 170 (59.0\%) of the controls were unemployed. The median income of the respondents was 250 ETB. The majority (364 (94.8\%)) of the study participants claimed they live far away from the health facility they receive the treatment from. "Car" was mentioned by $96(100 \%)$ of the cases and $268(93.1 \%)$ of the controls as a means of transportation to reach the health facility (see Table 1).

Table 1. Socio-demographic distribution of cases and controls, Addis Ababa, 2013.

\begin{tabular}{|c|c|c|c|c|}
\hline \multirow{2}{*}{\multicolumn{2}{|c|}{ Variables }} & \multicolumn{2}{|c|}{ Number of patients } & \multirow{2}{*}{ Total $(\%)(n=384)$} \\
\hline & & Cases $(\%)(n=96)$ & Controls $(\%)(n=288)$ & \\
\hline \multirow{2}{*}{ Sex } & Male & 55 (57.3\%) & $165(57.2 \%)$ & $220(57.2 \%)$ \\
\hline & Female & $41(42.7 \%)$ & $123(42.7 \%)$ & $164(42.7 \%)$ \\
\hline \multirow{4}{*}{ Age (years) } & $18-28$ & $30(31.3 \%)$ & 91 (31.6\%) & $121(31.5 \%)$ \\
\hline & $29-39$ & 35 (36.5\%) & 107 (36.5\%) & $142(36.2 \%)$ \\
\hline & $40-50$ & $22(22.9 \%)$ & $66(22.9 \%)$ & $88(22.9 \%)$ \\
\hline & +51 & $9(9.4 \%)$ & $24(9.0 \%)$ & $33(9.3 \%)$ \\
\hline \multirow{4}{*}{ Religion } & Orthodox & 70 (72.9\%) & $248(86.1 \%)$ & $318(82.8 \%)$ \\
\hline & Muslim & $8(8.3 \%)$ & $9(3.1 \%)$ & $17(4.4 \%)$ \\
\hline & Catholic & $0(0 \%)$ & $4(1.4 \%)$ & $4(1.0 \%)$ \\
\hline & Protestant & $18(18.8 \%)$ & 27 (9.4\%) & 45 (11.7\%) \\
\hline \multirow{3}{*}{ Level of Education } & Illiterate & $11(11.5 \%)$ & $67(23.3 \%)$ & $78(20.3 \%)$ \\
\hline & Primary education & $55(57.3 \%)$ & $82(28.5 \%)$ & 137 (35.7\%) \\
\hline & Secondary education and above & 30 (31.3\%) & $139(48.3 \%)$ & $169(44.0 \%)$ \\
\hline \multirow{2}{*}{ Employment status } & Employed & 19 (19.8\%) & $118(41.0 \%)$ & 137 (53.7\%) \\
\hline & Not employed & 77 (80.2\%) & $170(59.0 \%)$ & 247 (64.3\%) \\
\hline \multirow{3}{*}{ Monthly income (ETB) } & $<1000$ & $14(70 \%)$ & 77 (66.4\%) & $91(66.9 \%)$ \\
\hline & $1000-5000$ & $6(30.0 \%)$ & 39 (33.6\%) & 45 (33.1\%) \\
\hline & $>5000$ & $0(0 \%)$ & $0(0 \%)$ & $0(0 \%)$ \\
\hline \multirow{2}{*}{$\begin{array}{l}\text { Estimated distance of residence } \\
\text { from the health facility }\end{array}$} & Short & 2 (2.1\%) & 74 (25.7\%) & 76 (19.8\%) \\
\hline & Long & 94 (97.9\%) & $214(74.3 \%)$ & $308(80.2 \%)$ \\
\hline \multirow{2}{*}{$\begin{array}{l}\text { Means of transportation to } \\
\text { reach the health facility }\end{array}$} & On foot & $0(0 \%)$ & 20 (6.9\%) & $20(5.2 \%)$ \\
\hline & By car & 96 (100\%) & 268 (93.1\%) & 364 (94.8\%) \\
\hline \multirow{3}{*}{$\begin{array}{l}\text { Estimated time it takes to } \\
\text { reach the health facility (hrs) }\end{array}$} & $<1 \mathrm{hr}$ & $4(4.2 \%)$ & $111(38.5 \%)$ & 115 (29.9\%) \\
\hline & $1 \mathrm{hr}-24 \mathrm{hrs}$ & $76(79.2 \%)$ & $138(47.9 \%)$ & $214(55.7 \%)$ \\
\hline & $>24 \mathrm{hrs}$ & $16(16.7 \%)$ & 39 (13.5\%) & 55 (14.3\%) \\
\hline
\end{tabular}




\subsection{Factors Associated with MDR-TB}

There was a very wide previous TB treatment history difference between cases and controls in which 95 (99\%) of the cases were previously treated for TB while only 18 (6.3\%) of the controls had a previous TB treatment history. As a result, previous TB treatment (AOR $=5.7,95 \% \mathrm{CI}$ : $1.82-8.32$ ) was found to be a strong risk factor for MDR-TB. In addition, this study revealed having opportunistic infection, (AOR $=2.23,95 \%$ CI: 1.27 3.92), primary education ( $\mathrm{AOR}=1.37,95 \% \mathrm{CI}: 1.08$ - 1.74), unemployment (AOR = 3.44, 95\% CI: $1.82-6.48$ ), long distance of the health facility (AOR $=10.18,95 \%$ CI: $1.22-84.77), 1 \mathrm{hr}-24$ hrs to reach the health facility $(\mathrm{AOR}=2.19,95 \% \mathrm{CI}: 1.15-4.15)$ and $>24$ hrs to reach the health facility (AOR $=4.56,95 \% \mathrm{CI}: 1.14-18.25)$ as the most important factors contributing to the development of MDR-TB in Addis Ababa. However, this study did not indicate poor MDR-TB knowledge as a risk factor for MDR-TB, rather protective (see Table 2).

\section{Discussion}

Poor MDR-TB knowledge was not found as a risk factor for MDR-TB, rather protective, in contrary to the study done in China [16]. This might be due to the effective health education given to MDR-TB/HIV patients about their illness and because this study assessed their knowledge level after the health education was given. On the other hand, unemployment, long distance of the health facility, $1 \mathrm{hr}-24$ hrs to reach the health facility and $>24$ hrs to reach the health facility undoubtedly contribute to the rise of MDR-TB by limiting access to treatment and leading TB patients to default from anti-TB treatment. This finding was similar with studies done in China [16] and in Iran [17] which identified financial burden and unemployment as risk factors of MDR-TB. Moreover, Gler, M. T. et al. (2012) [18] demonstrated improving access to treatment for MDR-TB through decentralization of care to centers near the patient's residence reduced the risk of default $(0.3,95 \%$ CI: $0.2-0.7, P<0.001)$. Since default from treatment has a synergetic effect on spread of the disease it is to some extent consistent with this study's findings. In addition, this study found a strong association between previous treatment and MDR-TB similar to other studies [17]-[19]. This study also found having opportunistic infection, which is an indicator of

\section{Table 2. Factors associated with MDR-TB in multivariate analyses, Addis Ababa, 2013.}

\begin{tabular}{|c|c|c|c|}
\hline Variables & AOR & $95 \% \mathrm{CI}$ & $P$-value \\
\hline Previous TB treatment & 5.70 & $1.82-8.32$ & $<0.0001^{*}$ \\
\hline Poor knowledge & 0.003 & $0.0003-0.03$ & $<0.0001^{*}$ \\
\hline Having opportunistic infection & 2.23 & $1.27-3.92$ & $0.01^{*}$ \\
\hline Not having previous known contact with MDR-TB patient & 0.50 & $0.20-1.28$ & 0.15 \\
\hline Having BMI of 18.5 - 25 & 0.97 & $0.92-1.01$ & 0.15 \\
\hline Not having diabetes mellitus & 0.93 & $0.28-3.06$ & 0.90 \\
\hline Being muslim & 1.04 & $0.91-1.47$ & 0.80 \\
\hline Being protestant & 1.12 & $0.72-1.52$ & 0.77 \\
\hline Primary education & 1.37 & $1.08-1.74$ & $0.01^{*}$ \\
\hline Unemployment & 3.44 & $1.82-6.48$ & $0.0001^{*}$ \\
\hline Monthly income of 1000 - 5000 ETB & 0.66 & $0.12-3.52$ & 0.63 \\
\hline Long distance of the health facility & 10.18 & $1.22-84.77$ & $0.03^{*}$ \\
\hline $1 \mathrm{hr}-24 \mathrm{hrs}$ to reach the health facility & 2.19 & $1.15-4.15$ & $0.02^{*}$ \\
\hline$>24$ hrs to reach the health facility & 4.56 & $1.14-18.25$ & $0.03^{*}$ \\
\hline Drinking alcohol & 1.02 & $0.22-4.73$ & 0.98 \\
\hline Not defaulting from treatment & 0.86 & $0.45-1.63$ & 0.65 \\
\hline
\end{tabular}

Note: ${ }^{*}$ Factors significantly associated with MDR-TB $(P<0.05)$. 
immune-suppression, as a risk factor for MDR-TB. Similarly, a study done in four European countries revealed immune-suppression other than HIV as a risk factor for MDR-TB (OR 1.96) [20]. However, having primary education as a risk factor of MDR-TB was unexpected as education is perceived to enhance receptivity of information given. This somehow indicates the need to evaluate the teaching strategies used to determine how they influence assimilation of information given to people.

\section{Strength and Limitation of the Study}

One of the strength of this study was the design and having included all sub cities of Addis Ababa which made it a powerful representative study. Moreover, the sampling method and procedure used was targeted to decrease selection bias. The other strength of the study was being able to meet the minimum sample size requirement with in short study period even though finding MDR-TB/HIV cases happened to be rare. This may be because the clients were asked to participate by their service givers which minimized the non-response rate.

Even though this study provides important information about risk factors of MDR-TB, a number of limitations need to be considered. First, the study was prone to social desirability bias since the questions were administered by face to face interview. Absence of baseline data on the specific target population for comparison was also a problem. The other limitation was absence of a gold standard to measure knowledge level. Moreover, the study can only be generalized to PLW MDR-TB/HIV and TB/HIV in Addis Ababa.

\section{Conclusion and Recommendation}

In conclusion, previous TB treatment, having opportunistic infection, primary education, unemployment, long distance of the health facility, $1 \mathrm{hr}-24$ hrs to reach the health facility and $>24$ hrs to reach the health facility increase the risk of multi-drug resistance among PLW TB/HIV. Therefore, TB control programs under Addis Ababa health bureau should emphasize on these risk factors to control MDR-TB. In addition, the risk factors identified in this study need to be corroborated with multi-centre case-control studies to bring out nationally relevant risk factors for MDR-TB.

\section{Dedication}

"If I have seen further it is by standing on the shoulder of giants."-Isaac Newton-I dedicate this manuscript to Dr. Engineer Hirpa Lemu.

\section{References}

[1] Seung, K.J., Omatayo, D.B., Keshavjee, S., Furin, J.J., Farmer, P.E., et al. (2009) Early Outcomes of MDR-TB Treatment in a High HIV-Prevalence Setting in Southern Africa. PLOS ONE, 4, Article: e7186. http://dx.doi.org/10.1371/journal.pone.0007186

[2] Calver, A.D., Falmer, A.A., Murray, M., Strauss, O.J., Streicher, E.M., et al. (2010) Emergence of Increased Resistance and Extensively Drug-Resistant Tuberculosis despite Treatment Adherence, South Africa. Emerging Infectious Diseases, 16, 264-271. http://dx.doi.org/10.3201/eid1602.090968

[3] Hassim, S., Shaw, P.A., Sangweni, P., Malan, L., Ntshani, E., et al. (2010) Detection of a Substantial Rate of Multidrug-Resistant Tuberculosis in an HIV-Infected Population in South Africa by Active Monitoring of Sputum Samples. Clinical Infectious Diseases, 50, 1053-1059. http://dx.doi.org/10.1086/651119

[4] Gandhi, N.R., Shah, N.S., Andrews, J.R., Vella, V., Moll, A.P., et al. (2010) HIV Co-Infection in Multidrug- and Extensively Drug-Resistant Tuberculosis Results in High Early Mortality. American Journal of Respiratory and Critical Care Medicine, 181, 80-86. http://dx.doi.org/10.1164/rccm.200907-0989OC

[5] Dheda, K., Shean, K., Zumla, A., Badri, M., Streicher, E.M., et al. (2010) Early Treatment Outcomes and HIV Status of Patients with Extensively Drug-Resistant Tuberculosis in South Africa: A Retrospective Cohort Study. Lancet, 375, 1798-1807. http://dx.doi.org/10.1016/S0140-6736(10)60492-8

[6] WHO (2012) Tuberculosis Global Facts. www.who.int/tb

[7] Agonafi, M., et al. (2010) Phenotypic and Genotypic Analysis of Multidrug-Resistant Tuberculosis in Ethiopia. International Journal of Tuberculosis and Lung Disease, 14, 1259-1265.

[8] WHO (2010) Multidrug and Extensively Drug-Resistant TB (M/XDR-TB): Global Report on Surveillance and Response. 
[9] Demissie, M., Gebeyehu, M. and Berhane, Y. (2005) Primary Resistance to Anti-Tuberculosis Drugs. International Journal of Tuberculosis and Lung Disease, 1, 64-67.

[10] Abate, G., Miörner, H., Ahmed, O. and Hoffner, S.E. (2006) Drug Resistance in Mycobacterium Tuberculosis Strains Isolated from Re-Treatment Cases of Pulmonary Tuberculosis: Susceptibility to First-Line and Alternative Drugs. International Journal of Tuberculosis and Lung Disease, 2, 580-584.

[11] Meskel, D.W., Abate, G., Lakew, M., Goshu, S. and Aseffa, A. (2008) Anti-Tuberculosis Drug Resistance among Retreatment Patients. Ethiopian Medical Journal, 46, 219-225.

[12] Addis Ababa Regional Health Bureau (AARHB) (2010) Operational Manual for Regional HIV/AIDS Care and Treatment Catchments Team Activities.

[13] Ethiopian Federal Ministry of Health and Federal HIV/AIDS Prevention and Control Office (2007) Single Point HIV Prevalence Estimate.

[14] FMOH (2008) Tuberculosis, Leprosy and TB/HIV Prevention and Control Programme. Addis Ababa.

[15] Addis Ababa HIV/AIDS Prevention \& Control Office (2009) Anti-Retroviral Treatment (ART) Sites.

[16] Liang, L.B., Wu, Q.H., Gao, L.J., Hao, Y.H., Liu, C.J., Xie, Y.G., et al. (2012) Factors Contributing to the High Prevalence of Multidrug-Resistant Tuberculosis: A Study from China. Thorax, 67, e632-e638. http://dx.doi.org/10.1136/thoraxjnl-2011-200018

[17] Merza, M.A., et al. (2011) Anti-Tuberculosis Drug Resistance and Associated Risk Factors in a Tertiary Level TB Centre in Iran. Journal of Infection in Developing Countries, 5, 511-519. http://dx.doi.org/10.3855/jidc.1259

[18] Gler, M.T. (2012) Impact of Patient and Program Factors on Default during Treatment of Multidrug-Resistant Tuberculosis. International Journal of Tuberculosis and Lung Disease, 16, 955-960. http://dx.doi.org/10.5588/ijtld.11.0502

[19] Bushra, A.W. (2012) Risk Factors for Multi-Drug Resistant TB (MDR-TB) among Tuberculosis Patients in Saint Peter TB Specialized Hospital.

[20] Casal, M. (2005) A Case-Control Study for Multidrug-Resistant Tuberculosis: Risk Factors in Four European Countries. Microbial Drug Resistance, 11, 62-67. http://dx.doi.org/10.1089/mdr.2005.11.62

\section{Abbreviations and Acronyms}

TB: tuberculosis

MDR-TB: multi-drug resistant tuberculosis

XDR: extensively drug-resistant

ART: anti-retroviral therapy

HIV: human immuno-deficiency virus

PLW TB/HIV: people living with TB and HIV

BMI: body mass index

CI: confidence interval

ETB: ethiopian birr

COR: crude odds ratio

AOR: adjusted odds ratio

$\operatorname{Hr}(\mathrm{s})$ : hour(s) 